University of Wollongong

Research Online

Australian Institute for Innovative Materials -

Papers

Australian Institute for Innovative Materials

2010

A sign of field-induced first order magnetic state transition and giant reversible magnetocaloric effect in cobalt hydroxide nanosheets

Precious Shamba

University of Wollongong, ps807@uowmail.edu.au

Rong Zeng

University of Wollongong, rzeng@uow.edu.au

J Q Wang

University of Jinan, China

SX. Dou

University of Wollongong, shi@uow.edu.au

Follow this and additional works at: https://ro.uow.edu.au/aiimpapers

Part of the Engineering Commons, and the Physical Sciences and Mathematics Commons

Research Online is the open access institutional repository for the University of Wollongong. For further information contact the UOW Library: research-pubs@uow.edu.au 


\title{
A sign of field-induced first order magnetic state transition and giant reversible magnetocaloric effect in cobalt hydroxide nanosheets
}

\author{
Abstract \\ We report the synthesis of $\beta-\mathrm{Co}(\mathrm{OH})_{2}$ nanosheets using microwave assisted hydrothermal and \\ conventional chemical reaction methods. A magnetic transition at an onset temperature, $\mathrm{T}_{1} \sim 96 \mathrm{~K}$, and a \\ sign of antiferromagnetic state transition at the Néel temperature, $T_{N}=9 \sim 10 \mathrm{~K}$, can be determined from $\mathrm{M}$ - \\ $\mathrm{T}$ curves and $\mathrm{M}-\mathrm{H}$ curves magnetic properties investigation. It is found that a sign of field-induced first \\ order transition below $9 \mathrm{~K}$, act as a role of reversing the magnetocaloric effect from negative to positive \\ value. The large reversible magnetic-entropy change $\left(\Delta S_{M}\right)$ of $17 \mathrm{~J} / \mathrm{kg} \mathrm{K}$ around $11 \mathrm{~K}$ for a field change of \\ $5 \mathrm{~T}$ indicates that this material is useful for refrigeration applications at low temperatures.

\section{Keywords} \\ sign, field, induced, first, order, magnetic, state, transition, giant, reversible, magnetocaloric, effect, cobalt, \\ hydroxide, nanosheets

\section{Disciplines} \\ Engineering | Physical Sciences and Mathematics

\section{Publication Details} \\ Shamba, P, Zeng, R, Wang, JQ, \& Dou, SX (2010), A sign of field-induced first order magnetic state \\ transition and giant reversible magnetocaloric effect in cobalt hydroxide nanosheets, Journal of Applied \\ Physics, 107(9), pp. 1-3.
}




\title{
A sign of field-induced first order magnetic state transition and giant reversible magnetocaloric effect in cobalt hydroxide nanosheets
}

\author{
P. Shamba, ${ }^{1}$ R. Zeng, ${ }^{1, a)}$ J. Q. Wang, ${ }^{1,2}$ and S. X. Dou ${ }^{1}$ \\ ${ }^{1}$ Institute for Superconducting and Electronic Materials, University of Wollongong, \\ New South Wales 2522, Australia \\ ${ }^{2}$ School of Materials Science and Engineering, University of Jinan, Jinan 250022, \\ People's Republic of China
}

(Presented 19 January 2010; received 31 October 2009; accepted 12 January 2010; published online 21 April 2010)

We report the synthesis of $\beta-\mathrm{Co}(\mathrm{OH})_{2}$ nanosheets using microwave assisted hydrothermal and conventional chemical reaction methods. A magnetic transition at an onset temperature, $\mathrm{T}_{1}$ $\sim 96 \mathrm{~K}$, and a sign of antiferromagnetic state transition at the Néel temperature, $\mathrm{T}_{\mathrm{N}}=9 \sim 10 \mathrm{~K}$, can be determined from M-T curves and $\mathrm{M}-\mathrm{H}$ curves magnetic properties investigation. It is found that a sign of field-induced first order transition below $9 \mathrm{~K}$, act as a role of reversing the magnetocaloric effect from negative to positive value. The large reversible magnetic-entropy change $\left(\Delta S_{M}\right)$ of 17 $\mathrm{J} / \mathrm{kg} \mathrm{K}$ around $11 \mathrm{~K}$ for a field change of $5 \mathrm{~T}$ indicates that this material is useful for refrigeration applications at low temperatures. (C) 2010 American Institute of Physics. [doi:10.1063/1.3359813]

\section{INTRODUCTION}

Conventional refrigeration technologies based on the gas-compression/expansion cooling mechanisms may gradually be replaced by the environmentally friendly and more efficient magnetic refrigeration in the near future. ${ }^{1-5}$ The magnetic refrigeration is based on the magnetocaloric effect (MCE), which results from the coupling of a system of magnetic moments with an external magnetic field resulting in the cooling or heating of a system. An ideal material for magnetic refrigeration should be composed of relatively inexpensive raw materials, have a high MCE demonstrated by a high change in magnetic entropy $\left(\Delta \mathrm{S}_{\mathrm{M}}\right)$ and a high adiabatic temperature change $\left(\Delta \mathrm{T}_{\mathrm{ad}}\right)$, and have little or no thermal/magnetic hysteresis. ${ }^{6}$ First order transitions possess the abovementioned characteristics and have been shown to have a consistently higher MCE than second-order transitions. Recently, there has been an interest in the investigation of magnetic and MCE properties in various nanoparticle systems. ${ }^{7-10}$ Magnetic nanoparticles exhibit a higher refrigerant capacity as compared to their bulk counterparts, owing to their reasonably large MCE over a wide temperature range. ${ }^{11}$ It has now been established that the shapes of nanostructured materials have a considerable influence on their physical properties. ${ }^{12}$ In the past decade, one-dimensional nanostructures such as wires, rods, and tubes were the main focus of intense research, owing to their novel properties and potential applications in the fabrication of nanoscale devices. In recent years, however, there has been a growing interest in the investigation of two-dimensional (2D) nanosheets due to the interesting properties they possess, owing to their high anisotropy and thinness. ${ }^{13}$ Enhanced magnetic properties are also expected in 2D nanostructures. For $\beta-\mathrm{Co}(\mathrm{OH})_{2}$, this area is poorly explored, and to date, only a few reports on the

\footnotetext{
a) Author to whom correspondence should be addressed. FAX: 61-242215731. Electronic mail: rzeng@uow.edu.au.
}

preparation of 2D $\beta-\mathrm{Co}(\mathrm{OH})_{2}$ nanostructures have appeared in print. ${ }^{12,14}$ Liang et al. and Liu et al. both synthesized single crystalline $\beta-\mathrm{Co}(\mathrm{OH})_{2}$ nanosheets. However, these studies have not investigated the magnetic properties of the $2 \mathrm{D} \beta-\mathrm{Co}(\mathrm{OH})_{2}$ to establish whether the expected enhanced magnetic properties would be observed. To the best of our knowledge, this is the first report on the magnetic and magnetocaloric properties of $2 \mathrm{D} \beta-\mathrm{Co}(\mathrm{OH})_{2}$ nanostructures. Herein, we report a novel process of synthesizing $\beta-\mathrm{Co}(\mathrm{OH})_{2}$ nanosheets through a microwave assisted hydrothermal process. It is the aim of this work to investigate the magnetic and magnetocaloric properties of $\beta-\mathrm{Co}(\mathrm{OH})_{2}$ nanosheets and compare them with those of $\beta-\mathrm{Co}(\mathrm{OH})_{2}$ nanoparticles reported in literature.

\section{EXPERIMENTAL DETAILS}

Analytically pure reagents were used in this experiment. A precipitation and microwave hydrothermal process was used for the synthesis of cobalt hydroxide nanosheets. 10 mmol $\mathrm{Co}\left(\mathrm{NO}_{3}\right)_{2} \cdot 6 \mathrm{H}_{2} \mathrm{O}$ was dissolved in $50 \mathrm{ml}$ distilled water under stirring; then $2 M \mathrm{KOH}$ solution was added into the solution at a rate of $2 \mathrm{ml} \mathrm{min}^{-1}$, until the $p \mathrm{H}$ value of the solution reached 11 . This solution was stirred for $30 \mathrm{~min}$, after which it was transferred into a double-walled vessel, which consists of an inner liner and a cover made of Teflon polytetrafluoroethylene and an outer high strength sleeve. The vessel was sealed and maintained in a microwave accelerated reaction system (MARS-5, CEM Corporation, USA) at $140{ }^{\circ} \mathrm{C}$ for $3 \mathrm{~h}$. This system was operated at a frequency of $2.45 \mathrm{GHz}$ with a maximum power of $1200 \mathrm{~W}$ and could be controlled by temperature (maximum of $300{ }^{\circ} \mathrm{C}$ ) and pressure (maximum of $1500 \mathrm{psi}$ ). On completion of the reaction, the resultant black precipitates were centrifuged and washed with distilled water and then ethanol in an attempt to minimize the extra ions in the final product. Finally, the black precipitates were dried at $80{ }^{\circ} \mathrm{C}$ in air. 

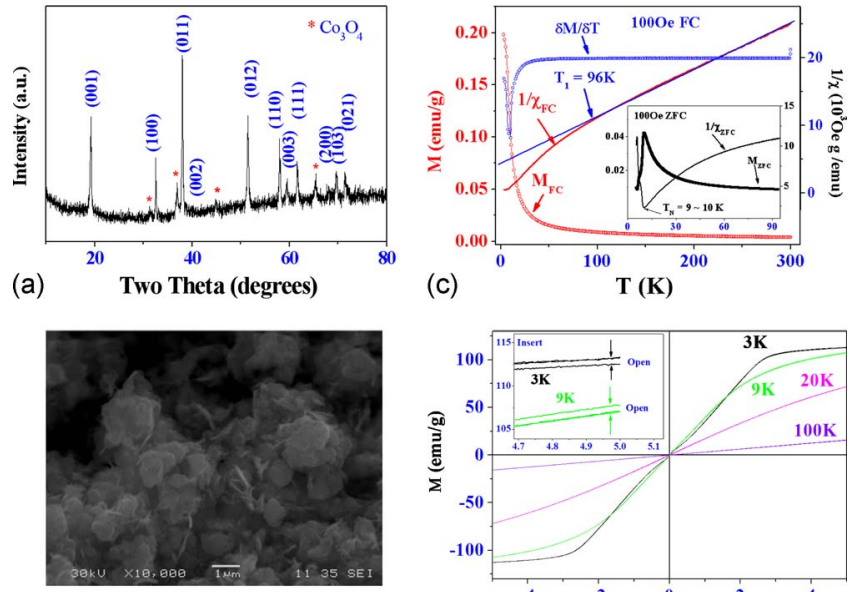

(b)

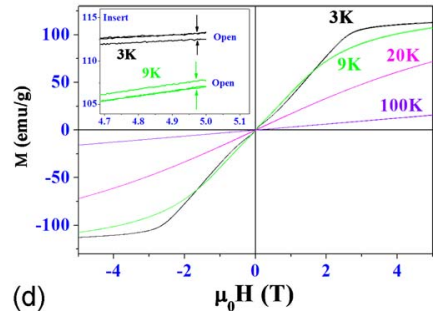

(d)

FIG. 1. (Color online) The XRD pattern and index (a) SEM image (b) and magnetic behaviors of cobalt hydroxide nanosheets, M-T, $1 / \chi-\mathrm{T}$, and $\delta \mathrm{M} / \delta \mathrm{T}-\mathrm{T}$ curves under $100 \mathrm{Oe} \mathrm{FC}$; the insert show the $\mathrm{M}-\mathrm{T}$ and $1 / \chi-\mathrm{T}$ curves under ZFC (c), selected M-H loops at temperatures of 3, 10, 20, and $100 \mathrm{~K}(\mathrm{~d})$.

The as-prepared sample was characterized by x-ray diffraction (XRD) and scanning electron microscopy (SEM). The magnetization measurements for the sample was carried out using the vibration sample magnetometer option of a quantum design $14 \mathrm{~T}$ physical property measurement system in the temperature range of $3-22 \mathrm{~K}$ at applied fields up to 5 T. The MCE was evaluated from the calculated isothermal magnetic entropy change $\Delta \mathrm{S}_{\mathrm{M}}(\mathrm{T}, \mathrm{H})$ in the vicinity of the magnetic phase transition temperature according to Eq. (1) below,

$$
\Delta \mathrm{S}_{\mathrm{M}}(\mathrm{T}, \mathrm{P}, \mathrm{H})_{\Delta \mathrm{H}, \mathrm{p}}=\int_{\mathrm{H}_{1}}^{\mathrm{H}_{2}}\left(\frac{\partial \mathrm{M}(\mathrm{T}, \mathrm{P}, \mathrm{H})}{\partial \mathrm{T}}\right)_{\mathrm{H}, \mathrm{p}} \mathrm{dH} .
$$

\section{RESULTS AND DISCUSSION}

The XRD pattern and index of $\mathrm{Co}(\mathrm{OH})_{2}$ sample were shown in Fig. 1(a); most of the $\beta-\mathrm{Co}(\mathrm{OH})_{2}$ phase $(\sim 95 \%)$ and a small amount of a secondary phase $\left(\sim 5 \% \mathrm{Co}_{3} \mathrm{O}_{4}\right)$ were determined through XRD Rietveld refinement. The morphology of the $\beta-\mathrm{Co}(\mathrm{OH})_{2}$ nanoparticles was investigated by SEM, as shown in Fig. 1(b). The $\beta-\mathrm{Co}(\mathrm{OH})_{2}$ is shown to have a platelet morphology with the diameter distribution range being 1-2 $\mu \mathrm{m}$. The platelet particle is consisted of agglomerated nanosheets with the average platelet thickness ranging between 20 and $80 \mathrm{~nm}$. However, the average thickness of a single $\beta-\mathrm{Co}(\mathrm{OH})_{2}$ nanosheet was about $10 \mathrm{~nm}$, estimated from the isolated nanosheets.

The temperature dependence of the inverse magnetic susceptibility in a magnetic field of 100 Oe under field cooling (FC) and zero FC (ZFC) procedures is shown in Fig. 1(c) (the insets show curves under ZFC conditions), which contains $1 / \chi-\mathrm{T}$ curves $\left(1 / \chi_{\mathrm{FC}}-\mathrm{T}\right.$ curve and $1 / \chi_{\mathrm{ZFC}}-\mathrm{T}$ curve $)$. A sign of antiferromagnetic (AFM) transition at temperature $\mathrm{T}_{\mathrm{N}}=9 \sim 10 \mathrm{~K}$ can be found from these $1 / \chi-\mathrm{T}$ curves. The $\mathrm{T}_{\mathrm{N}}$ value for $\beta-\mathrm{Co}(\mathrm{OH})_{2}$ nanoparticles in the present work is lower than the previously reported values of 11 and $12.3 \mathrm{~K}$
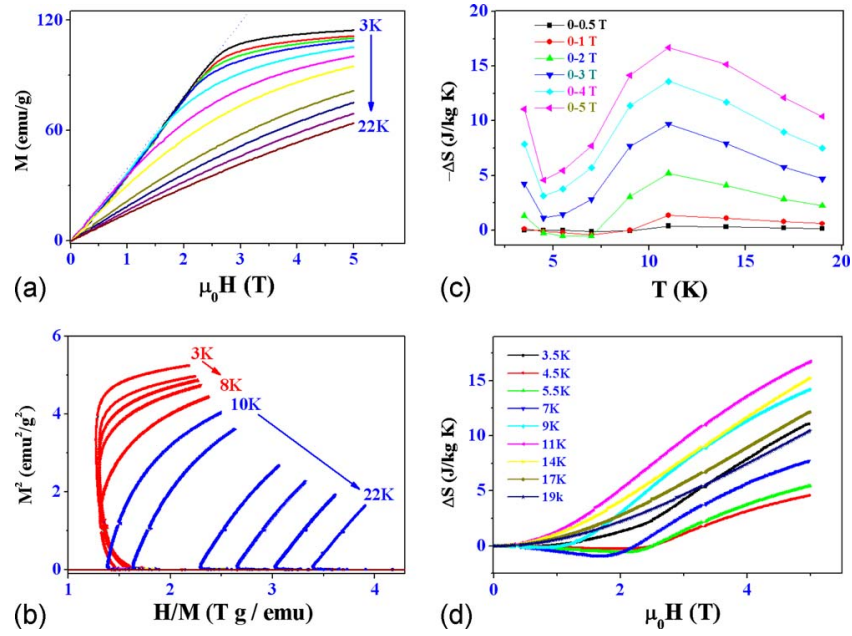

FIG. 2. (Color online) Magnetic behaviors of cobalt hydroxide nanosheets, $\mathrm{M}-\mathrm{H}$ curves at temperature from 3 to $22 \mathrm{~K}$ (a), Arrott-plot curves (b), magnetic-entropy changes verse temperature curves (c), and magneticentropy changes vs applied field (d).

reported in Refs. 15 and 16, respectively. A decrease in $T_{N}$ was observed in many nanosized AFM systems as the grain size decreased. ${ }^{17-19}$ We have established from the SEM images that the $\beta-\mathrm{Co}(\mathrm{OH})_{2}$ nanoparticles investigated in this work had a considerably smaller grain size, compared to those described in Refs. 15 and 16; hence the lower value of $\mathrm{T}_{\mathrm{N}}$ in the present work could be ascribed to nanosized effects. The $1 / \chi-\mathrm{T}$ curves also indicate that paramagnetic to spin glass (SG)-like (we suspect it is the surface spin present SG-like behavior) transition seems start at onset temperature defined as $\mathrm{T}_{1} \sim 96 \mathrm{~K}$ since the slope of the curve starts changing from the constant straight line (PM state) to lower value (SG-like state) at that temperature $(96 \mathrm{~K})$, then the value of slope increase (AFM state) at temperatures of 9 $\sim 10 \mathrm{~K}$. The SG-like behavior of the surface spins is clearly seen as an open hysteresis loop, as shown in the inset of Fig. 1(d). This indicates that it has a loss of magnetization during one hysteresis. ${ }^{20,21}$ Figure 1(d) shows the selected isothermal $\mathrm{M}-\mathrm{H}$ loops of the $\beta-\mathrm{Co}(\mathrm{OH})_{2}$ nanosheets, which are plotted as a function of the applied magnetic field (ranging from $\mu_{0} H=-5$ to $5 \mathrm{~T}$ ) at the temperatures of $3,10,20$, and $100 \mathrm{~K}$. This figure depicts the hysteresis loop of the $\beta-\mathrm{Co}(\mathrm{OH})_{2}$ nanosheets obtained at different temperatures in the presence of a magnetic field, which was scanned in the interval of \pm 5 $\mathrm{T}$. We found a magnetic hysteresis of approximately $20 \mathrm{Oe}$, which is very low. It should be noted that the $\mathrm{M}-\mathrm{H}$ loops show straight-type line (PM behavior) at temperatures higher than $100 \mathrm{~K}$, show bent-type line (SG-like behavior of the surface spin) at temperatures lower than $100 \mathrm{~K}$, and show step-type line (field-induced AFM to FM behavior) at temperatures lower than $9 \mathrm{~K}$.

Figure 2(a) shows the isothermal magnetization curves of the $\beta-\mathrm{Co}(\mathrm{OH})_{2}$ nanosheets, which are plotted as a function of the applied magnetic field (ranging from $\mu_{0} H=0$ to $5 \mathrm{~T}$ ) in the temperature range between 3 and $22 \mathrm{~K}$. It should be noted that at temperatures below $9 \mathrm{~K}$ and under a low field, the variation in the isotherm magnetizations with temperature is rather dramatic as compared to the isotherm mag- 
netizations at temperatures above $9 \mathrm{~K}$. However, under high magnetic field values, the variation in the isotherm magnetizations (in the whole temperature range) becomes gradual with no saturation being observed. This nonsaturation of the magnetization confirms the presence of a large anisotropy in the $\beta-\mathrm{Co}(\mathrm{OH})_{2}$ nanosheets. Also, the fact that the $\mathrm{M}-\mathrm{H}$ curves are nonsaturating with gradual curvature indicates superparamagnetic behavior.

The Arrott plots $\mathrm{M}^{2}$ versus $\mathrm{H} / \mathrm{M}$ at selected temperatures in the range of 3-22 $\mathrm{K}$ are shown in Fig. 2(b). Below the temperature of $9 \mathrm{~K}$, Fig. 2(b) shows S-shaped Arrott plots, which demonstrate that the transition temperature is around 9 $\mathrm{K}$ and the field-induced transition from the AFM to FM state is first order in nature.

The isothermal magnetization data was analyzed using the well known Maxwell relation shown in Eq. (1). The magnetic entropy changes $\left(\Delta \mathrm{S}_{\mathrm{M}}\right)$ versus temperature under different fields are shown in Fig. 2(c); $\Delta S_{M}$ was calculated from the isothermal curves according to Eq. (1) of the Maxwell relation, which is discussed in detail in Refs. 22-24. Figure $2(\mathrm{~d})$ shows the magnetic entropy change, $\Delta \mathrm{S}_{\mathrm{M}}$, as a function of applied filed at different temperatures. This positive $\Delta \mathrm{S}_{\mathrm{M}}$ indicates inverse MCE, which has also been previously observed in $\beta-\mathrm{Co}(\mathrm{OH})_{2}$ nanoparticles. ${ }^{15}$ The peak value of $\Delta \mathrm{S}_{\mathrm{M}}$ is $17 \mathrm{~J} / \mathrm{kg} \mathrm{K}$ at $11 \mathrm{~K}$ for a field change of $5 \mathrm{~T}$. This is slightly higher than the previously reported value of $\Delta \mathrm{S}_{\mathrm{M}}=15 \mathrm{~J} / \mathrm{kg} \mathrm{K}$ at $5 \mathrm{~T}$ for $\beta-\mathrm{Co}(\mathrm{OH})_{2}$ nanoparticles. $^{15} \mathrm{It}$ is also worth noting that the XRD analysis of our sample shows a small amount of a secondary phase $\left(\sim 5 \% \mathrm{Co}_{3} \mathrm{O}_{4}\right)$, which obviously has a detrimental effect on the determined value of $\Delta \mathrm{S}_{\mathrm{M}}$. This therefore means that we expect our samples to exhibit an even higher value of $\Delta S_{M}$ once their purity has been improved.

Following the above mentioned results and discussions, we find that (i) the SG-like surface spin may have a significant effect on the MCE (Ref. 11) and (ii) the sign of field-induced first order magnetic phase transition is possibly due to the result of the competition or interaction coupling of the surface spin, impure phase $\left[\mathrm{Co}_{3} \mathrm{O}_{4}\right.$ shown in Fig. 1(a)], and $\beta-\mathrm{Co}(\mathrm{OH})_{2}$ bulk magnetic behavior. We will continue to investigate the nanomagnetism of this material. The $\beta-\mathrm{Co}(\mathrm{OH})_{2}$ nanosheets, however, show a very high magnetic moment, the $\mathrm{M}_{\mathrm{s}} \sim 110 \mathrm{emu} / \mathrm{g}$ at $3 \mathrm{~K}$, as shown in Fig. 1(d), which causes a highly reversible giant MCE effect even with second transition at a temperature above $11 \mathrm{~K}$, is sign of field-induced first transition from the AFM to the FM below $9 \mathrm{~K}$, and acts as a role of reversing the MCE value from negative to positive value in the low field range.

\section{CONCLUSION}

In conclusion, $\beta-\mathrm{Co}(\mathrm{OH})_{2}$ nanosheets have been successfully prepared by a microwave assisted hydrothermal and conventional chemical reaction method. This material displays a high magnetic moment and a large reversible magnetic entropy change of $17 \mathrm{~J} / \mathrm{kg} \mathrm{K}$ at $11 \mathrm{~K}$ in a magnetic field change of $5 \mathrm{~T}$, thereby indicating the suitability of this material for use in magnetic refrigeration.

\section{ACKNOWLEDGMENTS}

This work was supported by the Australian Research Council through a Discovery project (Project No. DP0879070).

${ }^{1}$ K. A. Gschneidner, Jr., V. K. Pecharsky, and A. O. Tsokol, Rep. Prog. Phys. 68, 1479 (2005).

${ }^{2}$ A. M. Tishin and Y. I. Spichkin, Series in Condensed Matter (IOP, London, 2003).

${ }^{3}$ H. Wada and Y. Tanabe, Appl. Phys. Lett. 79, 3302 (2001).

${ }^{4}$ B. Li, J. Du, W. J. Ren, W. J. Hu, Q. Zhang, D. Li, and Z. D. Zhang, Appl. Phys. Lett. 92, 242504 (2008).

${ }^{5}$ J. Du, W. B. Cui, Q. Zhang, S. Ma, D. K. Xiong, and Z. D. Zhang, Appl. Phys. Lett. 90, 042510 (2007).

${ }^{6}$ K. A. Gschneidner, Jr. and V. K. Pecharsky, Annu. Rev. Mater. Sci. 30, 387 (2000).

${ }^{7}$ Y. Du and D. O'Hare, Inorg. Chem. 47, 3234 (2008).

${ }^{8}$ A. Nittke, P. Esquinazi, W. Widder, and H. F. Braun, Physica C 260, 273 (1996).

${ }^{9}$ S. Ma, W. B. Cui, D. Li, N. K. Sun, D. Y. Geng, X. Jiang, and Z. D. Zhang, Appl. Phys. Lett. 92, 173113 (2008).

${ }^{10}$ S. R. Ahmed, S. B. Ogale, G. C. Papaefthymiou, R. Ramesh, and P. Kofinas, Appl. Phys. Lett. 80, 1616 (2002).

${ }^{11}$ M. H. Phan, M. B. Morales, C. N. Chinnasamy, B. Latha, V. G. Harris, and H. Srikanth, J. Phys. D: Appl. Phys. 42, 115007 (2009).

${ }^{12}$ Z. H. Liang, Y. J. Zhu, G. F. Cheng, and Y. H. Huang, Can. J. Chem. 84, 1050 (2006).

${ }^{13}$ J. Q. Hu, Y. Bando, J. H. Zhan, Y. B. Li, and T. Sekiguchi, Appl. Phys. Lett. 83, 4414 (2003).

${ }^{14}$ X. Liu, R. Yi, N. Zhang, R. Shi, X. Li, and G. Qiu, Chem. Asian J. 3, 732 (2008).

${ }^{15}$ X. H. Liu, W. Liu, W. J. Hu, S. Guo, X. K. Lv, W. B. Cui, X. G. Zhao, and Z. D. Zhang, Appl. Phys. Lett. 93, 202502 (2008).

${ }^{16}$ T. Takada, Y. Bando, M. Kiyama, and H. Miyamoto, J. Phys. Soc. Jpn. 21, 2726 (1966).

${ }^{17}$ J. P. Chen, C. M. Sorenson, K. J. Klabunde, G. C. Hadjipanayis, E. Devilin, and A. Kostikas, Phys. Rev. B 54, 9288 (1996).

${ }^{18}$ C. D. Spencer and D. Schroeer, Phys. Rev. B 9, 3658 (1974).

${ }^{19}$ T. Ambrose and C. L. Chien, Phys. Rev. Lett. 76, 1743 (1996).

${ }^{20}$ M. Gruyters, Phys. Rev. Lett. 95, 077204 (2005).

${ }^{21}$ E. L. Salabaş, A. Rumplecker, F. Kleitz, F. Radu, and F. Schüth, Nano Lett. 6, 2977 (2006).

${ }^{22}$ V. K. Pecharsky and K. A. Gschneidner, Jr., J. Appl. Phys. 86, 565 (1999).

${ }^{23}$ V. K. Pecharsky and K. A. Gschneidner, Jr., J. Appl. Phys. 90, 4614 (2001).

${ }^{24}$ H. W. Zhang, J. Shen, Q. Y. Dong, T. Y. Zhao, Y. X. Li, J. R. Sun, and B. G. Shen, J. Magn. Magn. Mater. 320, 1879 (2008). 